CAHIERS DE

NARRATOLOGIE

\section{Cahiers de Narratologie}

Analyse et théorie narratives

33 | 2018

L'Art du roman chez Umberto Eco

\title{
Un village français. L'histoire au risque de la fiction de Bernard Papin
}

\section{Marc Marti}

\section{(2) OpenEdition}

\section{Journals}

Electronic version

URL: http://journals.openedition.org/narratologie/8523

DOI: $10.4000 /$ narratologie. 8523

ISSN: 1765-307X

Publisher

LIRCES

\section{Electronic reference}

Marc Marti, « Un village français. L'histoire au risque de la fiction de Bernard Papin », Cahiers de Narratologie [Online], 33 | 2018, Online since 23 July 2018, connection on 25 September 2020. URL http://journals.openedition.org/narratologie/8523 ; DOI : https://doi.org/10.4000/narratologie.8523

This text was automatically generated on 25 September 2020

Article L.111-1 du Code de la propriété intellectuelle. 


\title{
Un village français. L'histoire au risque de la fiction de Bernard Papin
}

\author{
Marc Marti
}

\section{REFERENCES}

Papin Bernard, Un village français. L'histoire au risque de la fiction, Paris, Atlande, 2017, 220 pages.

1 Les éditions Atlande ont créé la collection A suivre... dirigée par François Jost. Elle est consacrée aux séries culte de la télévision et compte à ce jour quatre essais, dont le dernier en date est celui de Bernard Papin, consacré à la série française Un village français, qui a été diffusée entre 2010 et 2017 sur France 2. Cette fiction télévisuelle narre la vie d'une bourgade franc-comtoise fictive pendant la seconde guerre mondiale, de l'arrivée des Allemands en 1940 jusqu'à la libération en 1944 (saisons 1 à 6). La septième saison se déroule en 1944-45, au moment de la mise en place des nouvelles autorités, tout en faisant des sauts dans le futur, principalement les années 70 et le début des années 2000. L'auteur, actuellement maître de conférences à l'université Paris Sud, est membre du CEISME-CIM (Centre d'Etudes sur les Images et les Sons Médiatiques-Communication Information Médias). Il est spécialiste de la représentation de l'Histoire à la télévision, ayant déjà publié ou dirigé plusieurs ouvrages sur le sujet, en particulier sur l'époque des Lumières ${ }^{1}$.

2 L'étude à proprement parler occupe 168 pages, illustrées par une trentaine de photogrammes en noir et blanc. Elle est complétée par une partie "Outils » d'une soixantaine de pages, composée par les fiches techniques des saisons et des épisodes, qui décrivent les équipes de scénaristes par saison et épisode, ainsi que la réalisation et les dates de première diffusion. Les pages finales proposent des extraits de l'entretien accordé par Frédéric Krivine, qui a été directeur de l'écriture de l'ensemble des saisons ${ }^{2}$. 
3 Le préambule pose d'emblée l'originalité du sujet dans le paysage français, à savoir une fiction qui s'est développé sur 7 saisons, soit 72 épisodes, ce qui est déjà remarquable et qui témoigne de l'engouement des spectateurs ( $11 \%$ d'audience stabilisée avec des maxima à $20 \%$ lors des premières saisons). Cet engouement était à interroger, à l'aune de la prise de risque que constituait le choix d'une thématique potentiellement explosive, soixante-dix ans après les faits historiques évoqués, à savoir ce que fut la seconde guerre mondiale pour la France : l'Occupation et la Libération, les éléments militaires passant au second plan. L'auteur dessine la stratégie d'écriture, qui a reposé sur une équipe de scénaristes renouvelée et évolutive, un conseiller historique et une conseillère psychologique arrivée au terme de la saison 2 pour la construction des personnages. Sur ce point, comme l'indique le directeur de l'écriture, le choix a été fait de privilégier la " condition humaine » par rapport à l'Histoire (ou dans son rapport à l'Histoire). Le préambule pose aussi la question théorique (récurrente dans les études universitaires) du rapport entre fiction et histoire et il y répond de façon pragmatique, en citant judicieusement les propos des créateurs.

4 Le premier chapitre, "D'un débarquement à l'autre ", analyse le traitement du temps choisi cette longue fiction. L'analyse du comportement des personnages montre que finalement, le propos n'a pas été de faire apparaître l'héroïsme, mais plutôt l'humanité : violence, cruauté, cynisme et égoïsme sont des défauts partagés par l'ensemble du "personnel de la fiction » à des degrés divers, apparaissant comme la face obscure de l'héroïsme, de l'humanité, de l'altruisme et de la compassion. Le choix scénaristique est bien celui d'une véracité historique, ancrée dans une dimension anthropologique forte. Il explique sans doute l'attachement des spectateurs à des personnages, qui deviennent extrêmement complexes et qui évoluent au fil des épisodes et des saisons.

Dans «Au fil des jours et des saisons", Bernard Papin rappelle la particularité temporelle de la série, en se posant la question du rapport entre le temps historique et sa représentation. La durée de diffusion correspond à quarante longs métrages de quatre-vingt-dix minutes, avec un tournage étalé sur huit ans. Cette dernière donnée implique que les acteurs incarnant les personnages ont réellement vieilli, de même que les enfants ont grandi. Les seuls recours au maquillage temporel s'effectuent pour les séquences «dans le futur » de la dernière saison. Sur ce dernier point, la série adopte, dans les six première saisons, une chronologie linéaire assez stricte, sans flash-back et flash-forward. L'auteur voit plusieurs explications dans ce choix peu innovant, opposé à celui de nombre de séries américaines, qui multiplient le mélange des temps au point parfois de perdre volontairement le spectateur dans la chronologie comme Lost. Il s'agirait de ne pas bousculer un public plutôt âgé, celui des séries de France 3. Cependant, le propos historique de la fiction suppose aussi une relation mimétique avec l'historiographie, dont la principale caractéristique narrative est le récit linéaire. Enfin, avec un ensemble de personnages à la psychologie complexe, la linéarité temporelle permet de bien comprendre (donc de rendre vraisemblable) les évolutions des caractères, le passage de collabo à résistant, que ce soit par opportunisme ou par conviction, dans un contexte historique déterminant. Cette transformation temporelle des personnages renvoie aussi à la France de cette époque, dont on peut ainsi saisir l'évolution et le basculement. Seule la saison sept, dont le propos est d'interroger la mémoire, rompt avec ce système en multipliant les flash-forward et flash-back. 
6 La question du temps, du rythme plus précisément, occupe « Un jour, un épisode ». Les références temporelles sont précisées au spectateur par l'incrustation de dates en début d'épisode. L'auteur fait remarquer que la continuité et l'unité sont privilégiées : chaque épisode comporte peu d'ellipses et se déroule généralement sur une seule journée. Généralement, la progression suit une tresse temporelle, qui permet de mélanger les moments vécus par des personnages différents dans des endroits différents. Quelques épisodes font exception, en particulier ceux qui se rapportent aux événements majeurs comme l'arrivée des Allemands en 1940 ou le défilé des maquisards du 11 novembre 1943.

7 "Une dramatisation qui s'accentue au fil des saisons » analyse l'ensemble des effets qui y contribuent. La densité dramatique s'accentue à partir de la saison deux, pour se poursuivre sur les saisons suivantes, qui tendent à concentrer l'intrigue sur des moments-clés de l'Histoire, en disposant les personnages autour de l'événement. Cette dramatisation va de pair avec le suspense qui est traité dans « La tension narrative et ses procédés». Celui-ci se retrouve à l'intérieur des épisodes, sur le principe de l'incertitude narrative, amenant le spectateur à se poser la question «que va-t-il se passer? ». L'effet maximum est obtenu sur les moments de cliffhanger soit une tension particulière forte, située en fin d'épisode. On retrouve là un des principes de l'écriture sérielle, qui vise à maintenir le spectateur dans son désir de continuer à suivre la fiction, bien que dans certains cas, le procédé ait été utilisé pour des épisodes diffusés les uns à la suite des autres. Bernard Papin revoit aussi de façon critique les moments où la surprise du spectateur est parfois traitée de façon stéréotypée, avec des coups de théâtre relativement invraisemblables (le retour de Kurt en 1944, en tankiste blessé par exemple).

8 Le second chapitre, «L'écriture de l'Histoire par la fiction » pose la question générale du genre historique sous la forme d'une fiction télévisuelle. "Le réalisme de la chronique » analyse les divers procédés mis en œuvre pour créer ce que l'on pourrait nommer une "vraisemblance véridique ". D'abord, Un village français traite le temps sous la forme de l'homochronie, signe de la volonté de reconstitution temporelle. La reconstitution des décors et détails d'époque obéit à ce souci, sans sombrer dans l'esthétisme muséal. On serait ici tenté d'avancer l'idée de reconstruction plutôt que de reconstitution, d'autant que les extérieurs ont été tournés dans plusieurs départements français, dont aucun ne correspond à la Franche Comté, qui est pourtant l'espace référentiel de la fiction.

Costumes, coiffures, maquillages, objets du quotidien ont fait l'objet d'un travail de documentation historique avant d'être recréés. Les armes utilisées sont d'époque, comme les véhicules. En ce qui concerne les désignations des personnages, ils reprennent les tendances de la période historique en termes de prénoms. Comme le note finement Bernard Papin, un certain nombre de patronymes, en plus de leur véridicité historique, remplissent aussi un rôle de prédestination, comme le préfet Servier, qui servira servilement. La reconstruction sociologique de l'époque est aussi soignée, avec les représentants distincts d'une élite économique et politique locale, qui occupent une place de choix dans la distribution des rôles, une place somme toute logique étant donné leur rôle historique entre 1940 et 1945. Enfin, la préoccupation alimentaire sous-tend, comme un leitmotiv la vie quotidienne, une partie des épisodes et on pourrait remarquer qu'elle occupe le devant de la scène dans quelques séquences consacrées aux trafics auxquels donne lieu le marché noir. 
10 «Comment l'histoire vient à la série » analyse les ancrages référentiels, qui sont finalement peu nombreux, que ce soit les personnages ou les lieux. Ce choix assumé par les créateurs est celui d'une fiction à hauteur des personnages ordinaires, qui servent par ailleurs souvent de "personnages point de vue ». C'est-à-dire que l'Histoire dans la fiction n'est pas un appendice savant et abstrait, artificiellement ajouté : elle est le quotidien des hommes et des femmes de Villeneuve. L'auteur donne en exemple les ocularisations (envahissement de la zone Sud) et les auricularisations, quand les informations sont données par la radio. Par « feintise historique », la série reprend un épisode s'étant réellement déroulé, celui du défilé des maquisards à Oyonnax le 11 novembre 1943, qui est transposé à Villeneuve, avec un certain nombre de détails identiques.

11 La problématique des personnages de fiction, en relation avec les personnages historiques, est aussi au centre de cette fiction. Comme l'analyse l'auteur, les créateurs, par la complexité psychologique des personnages, semblent ne pas avoir recours à une série de "personnages-types ». Cependant, les courants et les nuances politiques de l'époque sont représentées. L'exemple le plus développé est sans doute celui de Daniel Larcher, médecin et un temps premier magistrat du village. L'Histoire est en quelque sorte illustrée et portée par des humains.

12 Le troisième chapitre approfondit la question du personnage, indispensable pour faire fonctionner la fiction. Après un rappel théorique sur les différents processus de construction et d'identification, l'auteur indique que le personnage de série se différencie de celui de cinéma sur un point: sa "durée ». L'effet collatéral est celui d'une forte identification de l'acteur avec le personnage, qui vit au même rythme que le spectateur, ce qui lui confère une véritable consistance ontologique. Dans un village français, celle-ci s'épaissit dans le caractère des personnages, dont la plupart s'éloigne du prototype du héros ou du antihéros. Les personnages principaux sont ensuite analysés en détail dans ce chapitre, avec une interrogation légitime sur les bourreaux tels qu'ils sont représentés. Bernard Papin pose aussi la question de la relation du personnage avec le spectateur, où empathie et sympathie se mêlent à la pulsion scopique telle que l'a définie la psychanalyse et que l'a adaptée pour le cinéma Christian Metz.

Logiquement, le chapitre suivant pose la question du téléspectateur. Celle-ci peut être traitée statistiquement grâce aux nombreux instruments de mesure dont disposent les chaînes du service public. Il en ressort un taux de suivi et de satisfaction extrêmement élevé, malgré l'érosion naturelle d'une série de sept saisons. Les qualités artistiques et éducatives ont été plébiscitées, de même que la relation affective aux personnages. Autre élément intéressant, le spectateur modèle apparaît comme quelqu'un d'âgé - $2 / 3$ de l'audience a plus de soixante ans - et de cultivé. Sa connaissance de l'Histoire est un des supports de la fiction. En contrepartie, les erreurs historiques, bien que peu nombreuses, sont impitoyablement listées par ce public. Selon Bernard Papin, une des grandes qualités de cette fiction est que les auteurs n'ont pas créé de «suspense indigne ", en jouant sur la différence entre le savoir du téléspectateur, connaissant l'Histoire, et celui des personnages, qui ignorent l'issue de la guerre ou les pratiques des Allemands. Par exemple, la sortie des Juifs de Villeneuve, où ils avaient été un temps détenus, se fait de façon déchirante et sans aucun espoir, alors que les personnages ignorent encore à ce moment-là l'existence des camps d'extermination. 
L'épilogue pose une question intéressante, celle de l'écriture des séries, qui débutent sans « connaître » leur fin, mettant en échec la théorie narratologique de la causalité régressive. Le récit sériel est ainsi " émergent ", cherchant toujours à se prolonger dans la saison suivante, se caractérisant ainsi par l'infinitude. Cependant, la dimension historique d'Un village français supposait qu'une fin adviendrait au terme de l'ultime saison représentant l'année 1945.

Sur le plan matériel, une donnée à prendre en compte pour une collection qui comporte des illustrations, on appréciera la qualité de l'objet, proposé au format carré 16x16, couverture couleur et papier couché brillant, agréable à lire. Le seul bémol sur ce point concerne la marge gauche des pages impaires, qui s'avère un peu juste pour le confort de lecture après une centaine de pages.

En annonçant sur son catalogue la collection par la phrase «Pour les fans », l'éditeur positionne la publication comme de la vulgarisation scientifique. On peut considérer, au regard du résultat, que l'objectif est atteint: tout en conservant la rigueur universitaire dans un appareil de références qui savent se faire oublier, Bernard Papin a produit un ouvrage très accessible et agréable à lire. Le contenu séduira un large public, intéressé par la façon dont la télévision peut mettre en fiction l'Histoire, mais aussi sur la manière dont sont conduites l'écriture et la réalisation des séries et la façon dont elle influe sur la trame narrative. Sur le plan universitaire, l'ouvrage apporte une réflexion intéressante sur les rapports complexes entre l'histoire et sa fictionalisation, tant dans la dimension formelle que dans les débats que cela peut susciter. Le narratologue trouvera aussi son compte dans une étude qui manie avec aisance les outils forgés pour le cinéma, la littérature et la série télévisuelle, du traitement du temps et de l'espace à celui des personnages, en passant par les questionnements sur la réception et les contraintes de la création.

\section{NOTES}

1. Bernard Papin, Nicolas le Floch, un "expert» au temps des Lumières, dir. Geneviève Landié, Bernard Papin et Alain Sebbah, L'Harmattan-INA, collection «Les médias en actes », 2014, 300 pages ; ainsi que Images du siècle des Lumières à la télévision. Construction d'une culture commune par la fiction, dir., De Boeck-INA, collection Medias-recherche, 2010, 208 pages.

2. Entretien mené par l'auteur et François Jost et publié dans le numéro 8 de la revue Télévision. 


\section{AUTHOR}

\section{MARC MARTI}

Université Nice Côte d'Azur, LIRCES, EA3159 